\title{
Strange Quark Stars - A Review
}

\author{
R. X. Xu \\ School of Physics, Peking University, Beijing 100871, China
}

\begin{abstract}
.
A pedagogical overview of strange quark matter and strange stars is presented. After a historical notation of the research and an introduction to quark matter, a major part is devoted to the physics and astrophysics of strange stars, with attention being paid to the possible ways by which neutron stars and strange stars can be distinguished in astrophysics. Recent possible evidence for bare strange stars is also discussed.
\end{abstract}

\section{Historical notes}

Soon after the Fermi-Dirac form (in 1926) of statistical mechanics was proposed for particles which obey Pauli's exclusion principle (in 1925), Fowler (1926) realized that the electron degeneracy pressure can balance for those stars, called as white dwarfs, discovered by astronomers in 1914. By a numerical calculation (1931) for a polytropic gas of extremely relativistic electrons, Chandrasekhar found a unique mass, which was interpreted as a mass limit of white dwarfs. Landau (1932) presented an elementary explanation of the Chandrasekhar limit by considering the lowest total energy of stars, and recognized that increasing density favors energetically the formation of neutrons, discovered only several months before by Chadwick, through the action $p+e^{-} \leftrightarrow n+\nu_{\mathrm{e}}$. A very massive object with much high density may have almost neutrons in the chemical equilibrium, which was then called as neutron stars (NSs).

Detailed calculations of NS structures showed (e.g., Oppenheimer \& Volkoff 1939) that an NS can have a mass of $\sim 1 M_{\odot}$, but is only $\sim 10 \mathrm{~km}$ in radius, which makes it hard to be observed by astronomers. However, on one hand, a few authors do investigate possible astrophysical implications of NSs. For example, Baade \& Zwicky (1934) combined the researches of supernovae, cosmic rays, and NSs, and suggested that NSs may form after supernovae; Pacini (1967) even proposed that the stored energy in rotational form of an NS could be pumped into the supernova remnant by emitting electromagnetic waves. On the other hand, NS models were developed with improved treatments of equation of states, involving not only $\left\{n, p, e^{-}\right\}$, but also mesons and hyperons. The cooling behavior of NSs was also initiated in 1960s due to the discovery of X-ray sources which were at first though mistakenly to be the thermal emission from NSs.

The discovery of radio pulsars by Hewish \& Bell (and their coauthors 1968) is a breakthrough in the study, and this kind of stars were soon identified as spinning NSs by Gold (1968). Since then more and more discoveries in other wave bands broaden greatly our knowledge about these pulsar-like compact stars 
(PLCSs), including X-ray pulsars, X-ray bursts, anomalous X-ray pulsars, soft $\gamma$-ray repeaters, and ROSAT-discovered "isolated neutron stars". It is still a current concept among astrophysicists that such stars are really NSs. NS studies are therefore in two major directions: 1, the emission mechanisms for the stars, both rotation-powered and accretion-powered; 2, the NS interior physics.

However, neutrons and protons are in fact not structureless points although they were thought to be elementary particles in 1930s; they (and other hadrons) are composed of quarks proposed by Gell-Mann and Zweig, respectively, in 1964. The quark model for hadrons developed effectively in 1960s, and Ivanenko \& Kurdgelaidze (1969) began to suggest a quark core in massive compact stars. Itoh (1970) speculated about the exist of 3-flavor full quark stars (since only $u, d$ and $s$ quarks were recognized at that time), and even calculated the hydrostatic equilibrium of such quark stars which are now called as strange stars (SSs). Is it possible that strange stars really exist in nature? The possibility increases greatly if the Bodmer-Witten's conjecture is correct: Bodmer (1971) initiated the discussion of quark matter with lower energy per baryon than normal nucleus, whereas Witten (1984) considered an assumption of stable 3-flavor quark matter in details and discussion extensively three aspects related $(1$, dark baryon and primordial gravitational wave due to the cosmic separation in the QCD epoch; 2, strange quark stars; 3, cosmic rays). Farhi \& Jaffe's (1984) calculation in the MIT bag model showed that the energy per baryon of strange matter is lower than that of nucleus for QCD parameters within rather wide range although we can hardly prove weather the Bodmer-Witten's conjecture is correct or not from first principles. Haensel, Zdunik \& Schaeffer (1986) and Alcock, Farhi \& Olinto (1986) then modelled SSs, and found that SSs can also have typical mass (of $\sim 1-2 M_{\odot}$ ) and radius (of $\sim 10 \mathrm{~km}$ ), which mean that the pulsar-like compact stars believed previously to be NSs might actually be SSs.

Yet the most important and essential thing in the study is: how to distinguish SSs from NSs observationally? More and more SS candidates appeared recently in literatures (e.g., Bombaci 2002, Xu 2002). It is generally suggested that SSs as radio pulsars, the most popular ones of PLCSs, should have crusts (with mass $\sim 10^{-5} M_{\odot}$ ) being similar to the outer crusts of NSs (Witten 1984, Alcock et al. 1986). But this view was criticized by Xu \& Qiao (1998), who addressed that bare strange stars (BSSs, i.e., SSs without crusts) being chosen as the interior of radio pulsars have three advantages: 1, the spectral features; 2 , the bounding energy; and 3, the core collapse process during supernova. It is thus a new window to distinguish BSSs from NSs via their magnetosphere and surface radiation according to the striking differences between the exotic quark surfaces of BSSs and the normal matter surfaces of NSs. With regard to the possible methods of finding strange stars in literatures, hard evidence to identify a strange star may be found by studying only the surface conditions since the other avenues are subject to many complex nuclear and/or particle physics processes that are poorly known. Thanks to those advanced X-ray missions, it may be a very time for us to identify real strange stars in the universe.

It is worth mentioning that, though some authors may name a general term "neutron star", regardless of that the stars are "neutron" or "strange", it is actually not suitable to call a real SS to be an NS since no neutron in an SS. 


\section{The standard model of particle physics and the quark matter}

One of the most great achievements in the last century is the construction of the standard model in particle physics (e.g., Cottingham \& Greenwood 1998), which asserts that the material in the universe is made up of elementary fermions (divided into quarks and leptons) interacting though gauge bosons: photon (electromagnetic), $\mathrm{W}^{ \pm}$and $\mathrm{Z}^{0}$ (weak), 8 types of gluons (strong), and graviton (gravitational). There are totally 62 types of "building blocks" in the model. Besides the 13 types of gauge bosons, there are three generations of fermions (1st: $\left\{\nu_{\mathrm{e}}\right.$, $\mathrm{e} ; \mathrm{u}, \mathrm{d}\}, 2 \mathrm{nd}:\left\{\nu_{\mu}, \mu ; \mathrm{c}, \mathrm{s}\right\}$, and 3rd: $\left\{\nu_{\tau}, \tau ; \mathrm{t}, \mathrm{b}\right\}$. Note that each types of quarks has three colors) and their antiparticles. The final one, which is still not discovered, is the Higgs particle that is responsible to the origin of mass.

It is a first principle in the Yang-Mills theory that an interaction satisfies a corresponding local gauge symmetry, sometimes being broken spontaneously due to vacuum phase transition. The gauge theory for electromagnetic and weak interactions is very successful, with a high precision in calculation; whereas one can treat gravity using Einstein's general relativity theory if the length scale is not as small as the Plank scale $\left(\sim 10^{-33} \mathrm{~cm}\right)$ although a gauge theory of gravity is still not successful. As for the gauge theory for strong interaction, the quantum chromodynamics (QCD), however, is still developing, into which many particle physicists are trying to make efforts.

Nonetheless, QCD has two general properties. For strong interaction in small scale $(\sim 0.1 \mathrm{fm})$, i.e., in the high energy limit, the interacting particles can be treated as being asymptotically free; a perturbation theory of QCD (pQCD) is possible in this case. Whereas in larger scale $(\sim 1 \mathrm{fm})$, i.e., in the low energy limit, the interaction is very strong, which results in color confinement. The pQCD is not applicable in this scale (many non-perturbative effects appear then), and a strong interaction system can be treated as a system of hadrons in which quarks and gluons are confined. In this limit, we still have effective means to study color interaction: 1, the lattice formulation (LQCD), with the discretization of space-time and on the base of QCD, provide a non-perturbative framework to compute numerically relations between parameters in the standard model and experiments by first principles; 2, phenomenological models, which rely on experimental date available at low energy density, are advanced for superdense hadronic and/or quark matter.

These two properties result in two distinct phases (Rho 2001) of hadronic matter, depicted in the QCD phase diagram in terms of temperature $T$ vs. baryon chemical potential $\mu_{\mathrm{B}}$ (or baryon number density). Hadron gas phase locates at the low energy-density limit where both $T$ and $\mu_{\mathrm{B}}$ are relatively low, while a new phase called quark gluon plasma (QGP) or quark matter appears in the other limit when $T$ or $\mu_{\mathrm{B}}$ is high although this new state of matter is still not found with certain yet. It is therefore expected that there is a kind of phase transition from hadron gas to QGP (or reverse) at critical values of $T$ and $\mu_{\mathrm{B}}$. Actually a deconfinement transition is observed in numerical simulations of LQCD for zero chemical potential $\mu_{\mathrm{B}}=0$, when $T \rightarrow T_{\mathrm{c}} \simeq(150 \sim 180) \mathrm{MeV}$.

Can we find real quark matter? Certainly we may improve very much the knowledge about the strong interaction by studying the matter's various properties if a QGP state is identified in hand. One way is to create high energy-density fireball in laboratory through the collisions of relativistic heavy 
ions in accelerators. Quark matter is expected if the center of the fireball reaches a temperature of $T_{\mathrm{c}}$, but the QGP is hadronized soon and there are final states of hadrons detected. It is thus a challenge to find clear signatures of QGP without ambiguousness in these experiments. Another way is to search celestial bodies which contain quark matter via cosmological or stellar processes. Strange stars (§3) are very probably such kind of objects, which are a kind of bulk QGP with mass $\sim 1 M_{\odot}$, composed of nearly equal number of up, down, and strange quarks (called as strange quark matter, or strange matter). Some possible observational signatures of SSs are also addressed in literatures. The first way to detect this new form of matter is in terrestrial laboratory physics (lab-physics), whereas the second is in astrophysics. They compensate each other. Up to date, the research both in lab-physics and in astrophysics faces a general difficulty in finding quark matter: to search a clear signature for its existence.

In fact, the investigations in lab-physics and astrophysics are in two different regions in the QCD phase diagram. Available Monte Carlo simulations of LQCD are only applicable for cases with $\mu_{\mathrm{B}}=0$, and thus give valuable guidance for labphysics. However, it is a very different story with SSs, where the density effects dominate $\left(\mu_{\mathrm{B}} \neq 0\right)$. It turns out, for technical reasons, to be extremely difficult to study strange matter by LQCD, and we have to rely on phenomenological models to speculate on the properties of SSs by extrapolating our knowledge at nuclear matter density. For an SS, with high $\mu_{\mathrm{B}}$ but low $T$, one can not naively think that it is a simple QGP; in fact many interesting phenomena, e.g., color superconductivity (Alford et al. 2001) of strange matter, are discussed. In this meaning, SSs provide only examples to study such hadronic system at high density, and one thus has to learn "experimental date" from astrophysics.

\section{Strange stars}

That strange stars (rather than neutron stars) are residual after core-collapse type supernova explosion depends upon 1, quark deconfinement occurs, 2 , strange matter in bulk is absolutely stable (Bodmer-Witten's conjecture). Unfortunately no certain answer to these issues is obtained theoretically $(\S 2)$. Nevertheless, we still can obtain general concepts about those two requirement for forming SSs by following simple arguments. In the view of bag model ${ }^{1}$, the vacuum in and out hadrons is different; in-hadron is of "pQCD vacuum" where the strong interaction is weak and pQCD is applicable, but out-hadron is of "QCD vacuum" where the nonlinear strong coupling between quarks or gluons is in control. As baryon number density $\varrho$ gets higher and higher, the pQCD vacuum in nucleons becomes more and more dominated; if nucleon keeps a radius $r_{\mathrm{n}} \sim 1 \mathrm{fm}$, the QCD vacuum disappears when $\varrho>\varrho_{\mathrm{c}} \sim\left(4 \pi r_{\mathrm{n}}^{3} / 3\right)^{-1} \sim 1.5 \varrho_{0}\left(\varrho_{0}\right.$ is the density of ordinary nuclear matter). Depending on rotation frequency and stellar mass, such density are easily surpassed in the cores of neutron stars (Weber 1999). Therefore the first requirement - quark deconfinement - may be satisfied. In case of $\varrho>\varrho_{\mathrm{c}}, u$ and $d$ quarks deconfined from nucleons could have a Fermi

\footnotetext{
${ }^{1}$ Quarks are though as degenerate Fermi gases in the model, which exist only in a region of space endowed with a vacuum energy density $B$ (called as the "bag constant").
} 
energy $^{2}>\sim 400 \mathrm{MeV}$, which is much larger than the mass of $s$ quark $m_{\mathrm{s}} \sim 150$ $\mathrm{MeV}$; the system should be energetically favorable by opening of a third flavor degree of freedom ( $s$ quark) since high-energy $u$ and $d$ quarks are expected to decay into $s$ quarks via weak interactions. The second requirement may thus also be reasonable. It is worth addressing that, if only the first requirement is satisfied while strange matter is not absolutely stable but only metastable, a mixed phase where bulk quark and nuclear matter could coexist over macroscopical distances may appear in NS cores (such NSs are called as "mixed stars", see, e.g., Heiselberg \& Hjorth-Jensen 2000, for a review).

These two requirements may lead to the existence of strange matter in nature. In a simplified version of the bag model, assuming quarks are massless and noninteracting, we then have quark pressure $P_{\mathrm{q}}=\rho_{\mathrm{q}} / 3\left(\rho_{\mathrm{q}}\right.$ is the quark energy density); the total energy density is $\rho=\rho_{\mathrm{q}}+B$ but the total pressure is $P=P_{\mathrm{q}}-B$. One therefore have the equation of state for strange matter,

$$
P=(\rho-4 B) / 3 \text {. }
$$

(Actually strange matter may have baryons from several hundreds ${ }^{3}$, called as strangelets, to about that of our sun, called as strange stars.) One can obtain the mass $M$ and radius $R$ of an SS by integrating numerically the TOV equation, with a strange matter equation of state [e.g., eq.(1)], assuming a certain central density (or pressure). The mass-radius relations of SSs calculated with various strange matter equations of states are very different from that of NSs, which may provide a possible way to differentiate SSs from NSs (§3.2). But an SS usually can have a mass of $1 \sim 2 M_{\odot}$, and correspondent radius $\sim 10 \mathrm{~km}$.

It is worth noting that the apparent temperature $T_{\infty}$ and radius $R_{\infty}$ (also called as radiation radius, defined by $L_{\infty}=4 \pi R_{\infty}^{2} \sigma T_{\infty}^{4}$, where $L_{\infty}$ is the apparent luminosity at infinity and $\sigma$ is the Stefan-Boltzmann constant), i.e., the values observed at infinity, is not that observed at the stellar surface $(R$ is also the

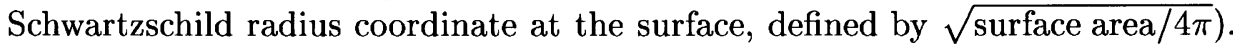
For a compact star with mass $M$ and radius $R$, the relations are (Haensel 2001) $T_{\infty}=T \sqrt{1-\left(R_{\mathrm{s}} / R\right)}, R_{\infty}=R / \sqrt{1-\left(R_{\mathrm{S}} / R\right)}$, where $R_{\mathrm{s}}=2 G M / c^{2}$ is the Schwartzschild radius.

\subsection{Structures: crusted or bare?}

As the strange quarks are more massive $\left(m_{\mathrm{s}} \sim 150 \mathrm{MeV}\right)$ than the up $\left(m_{\mathrm{u}} \sim 5\right.$ $\mathrm{MeV})$ and down $\left(m_{\mathrm{d}} \sim 10 \mathrm{MeV}\right)$ quarks, some electrons are required to keep the chemical equilibrium of an SS. This brings some interesting properties near the bare quark surface. Since quarks can be bound through strong interaction, whereas electrons by much weaker interaction (only the electromagnetism), the electrons can thus spread out the quark surface and be distributed in such a way that a strong outward static electric field is formed. Adopting a simple

\footnotetext{
${ }^{2}$ In the view of extremely relativistic ideal Fermi gases, one nucleon (mainly neutron) contributes about $1 u$ quark and $2 d$ quarks, and the Fermi energy $\varepsilon_{\mathrm{F}}=\left(3 \pi^{2}\right)^{1 / 3} \hbar c \cdot n^{1 / 3}$ ( $n$ the number density of quarks). If $\varrho=1.5 \varrho_{0}, \varepsilon_{\mathrm{F}}=379 \mathrm{MeV}$ for $u$ quark and $\varepsilon_{\mathrm{F}}=477 \mathrm{MeV}$ for $d$ quark.

${ }^{3}$ Strange matter may have a minimum mass limit due to the surface effects not being negligible, even if Bodmer-Witten's conjecture is correct.
} 
Thomas-Fermi model, one can deduce analytical expressions for the electron number density $n_{\mathrm{e}}$ and the electric field $E$ above the surface (Xu \& Qiao 1999),

$$
n_{\mathrm{e}} \sim \frac{9.5 \times 10^{35}}{\left(1.2 z_{11}+4\right)^{3}} \mathrm{~cm}^{-3}, \quad E \sim \frac{7.2 \times 10^{18}}{\left(1.2 z_{11}+4\right)^{2}} \mathrm{~V} \mathrm{~cm}^{-1}
$$

where $z$ is a measured height above the quark surface, $z_{11}=z /\left(10^{-11} \mathrm{~cm}\right)$. Very strong electric field, $\sim 10^{17} \mathrm{~V} / \mathrm{cm}$ from eq.(2), should be near the quark surface, which makes it possible to support a normal-matter crust with mass $\sim 10^{-5} M_{\odot}$ (Alcock et al. 1986). SSs covered by such crusts are called as crusted strange stars (CSSs), while SSs without crusts as bare strange stars (BSSs).

Can radio pulsars, the largest population of PLCSs, be BSSs? The answer was no in Alcock et al's (1986) paper: "Pulsar emission mechanisms which depend on the stellar surface as a source of plasma will not work if there is a bare quark surface" and "The universe is a dirty environment and a bare strange star may readily accrete some ambient material". Their first point is certainly incomplete, because $e^{ \pm}$pairs produced rapidly in strong electro-magnetic fields [as expressed in eq.(2), or induced by the unipolar effect] should create a corotating magnetosphere although no charged particles can be pulled out into the magnetosphere (Xu \& Qiao 1998). A nascent protostrange star should be bare because of strong mass ejection and high temperature (Usov 1998) after the supernova detonation flame; an SS can hardly accrete due to rapid rotating and strong magnetic field ( $\mathrm{Xu}$ et al. 2001); even accretion is possible, a crust still can not form as long as the accretion rate is not much larger than the Eddinton one ( $\mathrm{Xu}$ 2002). Their second point is thus also not reasonable. Therefore radio pulsars could be BSSs. Furthermore, it is found that BSSs may be better for explaining the observations of radio pulsars as well as other PLCSs (§3.3).

\subsection{Potential ways to identify a strange star}

Although the SS idea is not new, SS identification becomes a hot topic only in recent years because of advanced techniques in space. Since SSs may have similar masses and radii, which are conventional quantities observable in astronomy, to that of NSs, it is very difficult to find observational signals of quark matter in the PLCSs. It was argued that the SS and NS cooling behaviors could be distinguishable, since SSs may cool much faster than NSs (e.g. Pizzochero 1991). However, recent more complete analyses on this issue indicate that this may be impossible except for the first $\sim 30$ years after their births (Schaab et al. 1997). Nevertheless, the author thinks there may still be three effective ways to do.

1. The minimum rotation periods of SSs are smaller than that of NSs. Rotating stars composted of ideal fluid are subject to rotation-mode instability, which leads to the loss of rotation energy by gravitational radiation and results in substantial spindown. However the matter of a real star is not ideal but have viscosity; the calculated bulk viscosity, based on the work of Wang \& Lu (1984), of strange matter is much higher than that of neutron matter although their shear viscosities are similar; therefore SSs could have smaller periods at which their higher viscosity can prevent them from developing the instability (Madsen 1998, 2000). The $2.14 \mathrm{~ms}$ optical source in SN 1987A (Middleditch et al 2000) should be an SS if being confirmed in further observations. 
2. The approximate mass-radius $(M-R)$ relations of SSs $\left(M \propto R^{3}\right)$ are in surprising contrast to that of NSs $\left(M \propto R^{-3}\right)$, and SSs can have much small radii. Comparisons of observation-determined relations in X-ray binaries with modelled ones may thus tell if an object is an NS or an SS (Li et al. 1999). Also, a PLCS with radius $\$ 8 \mathrm{~km}$ could be an SS (Drake et al. 2002).

3. There are striking differences between the surfaces of BSSs and that of NSs. The very properties of the quark surface, e.g., strong bounding of particles, abrupt density change from $4 B \sim 4 \times 10^{14} \mathrm{~g} / \mathrm{cm}^{3}$ to $\sim 0$ [eq. (1)] in $\sim 1 \mathrm{fm}$, and strong electric fields, may eventually help us to identify of a BSS (§3.3).

\subsection{Observational evidence for bare strange stars?}

Three parts of possible evidence for BSSs are discussed below.

Although pulsar emission mechanism is not well understood, the RS-type (Ruderman \& Sutherland 1975) sparking model is still the popular one to connect magnetospheric dynamics with general observations, with an "user friendly" nature. Maybe the strongest support to the RS-type vacuum gap model is the drifting subpulses observed from some pulsars. However RS model faces at least two difficulties for NSs: the binding energy problem and the antipulsar issue, which can be solved completely if radio pulsars are BSSs (Xu et al. 1999).

The soft $\gamma$-ray burst of SGR 0526-66, with peak luminosity $\sim 10^{7} L_{\text {Edd }}$, needs ultra-strong field $\left(\sim 10^{17} \mathrm{G}\right)$ to constrain the fireball. An alternative bounding is through the quark surface; and it may be natural to explain the bursting energy and the light curves in a framework that a comet-like object falls to a BSS (Zhang et al. 2000, Usov 2001).

BSSs are expected to have featureless spectra (of both surface thermal and magnetospheric non-thermal components) since no ion is above the quark surface or in the magnetosphere, except for electron cyclotron lines due to the Landau levels appeared in strong fields (Xu \& Qiao 1998, Xu 2002). Recent observations known hitherto of several PLCSs actually show featureless spectra except for two sources 1E1207 and SGR1806, the lines of which may originate from Landau level transitions in suitable field strength for the space facilities (astro-ph/0207079).

\section{Conclusions}

The theoretical bases of SSs are, to some extent, solid in physics and the formation of strange matter stars is possible in astrophysics; SSs could thus exist. Although each of the observed phenomena from PLCSs may be interpreted under the NS regime with unusual or artificial physical properties, it could be a natural way to understand the observations by updating NSs with SSs.

Acknowledgements. I wish to thank Profs. Ignazio Bombaci, Chongshou Gao, Naoki Itoh, Jes Madsen, and Vladimir Usov for discussions.

\section{References}

Alcock, C., Farhi, E., \& Olinto, A. 1986, ApJ, 310, 261

Alford, M., Bowers, J. A., Rajagopal, K. 2001, in Lecture Notes in Physics, 578, Physics of Neutron Star Interiors, ed. D. Blaschke, et al. (Springer), 235 
Baade, W., \& Zwicky, F. 1934, Phys. Rev., 45, 138

Bodmer, A. R. 1971, Phys. Rev., D4, 1601

Bombaci, I. 2002, invited talk, Compacta stars in the QCD phase diagrams, Copenhagen (Denmark), eConf C010815, 29 (astro-ph/0201369)

Chandrasekhar, S. 1931, ApJ, 74, 81

Cottingham, W. N., \& Greenwood, D. A. 1998, An introduction to the standard model of particle physics, Cambridge Univ. Press

Drake, J. J., et al. 2002, ApJ, 572, 996

Farhi, E., \& Jaffe, R. L. 1984, Phys. Rev., D30, 2379

Fowler, R. H. 1926, MNRAS, 87, 114

Gold, T. 1968, Nature, 218, 731

Haensel, P. 2001, A\&A, 380, 186

Haensel, P., Zdunik, J. L., \& Schaeffer, R. 1986, A\&A, 160, 121

Heiselberg, H., Hjorth-Jensen, M. 2000, Phys. Rep., 328, 237

Hewish, A., Bell, S.J., Pilkington, et al. 1968, Nature, 217, 709

Itoh N. 1970, Prog. Theor. Phys., 44, 291

Ivanenko, D., \& Kurdgelaidze, D. F. 1969, Lett. Nuovo Cimento, 2, 13

Landau, L. D., 1932, Phys. Z. Sowjetunion, 1, 285 (an expanded discussion of his ideal can be found in one of their courses "Statistical Physics".)

Li, X. D., et al. 1999, Phys. Rev. Lett., 83, 3776

Madsen, J. 1998, Phys. Rev. Lett., 81, 16

Madsen, J. 2000, Phys. Rev. Lett., 85, 10

Middleditch, J. et al. 2000, New Astronomy, 5, 243

Oppenheimer, J. R., \& Volkoff, G. M. 1939, Phys. Rev., 55, 374

Pacini, F. 1967, Nature, 216, 567

Pizzochero, P. M. 1991, Phys. Rev. Lett., 66, 2425

Rho, M. 2001, in AIP Conf. Proc., 556, Explosive phenomena in astrophysical compact objects, ed. H.-Y. Chang et al. (New York: AIP), 160

Ruderman, M.A., \& Sutherland, P.G. 1975, ApJ, 196, 51

Schaab, C., Hermann, B., Weber, F., \& Weigel, M.K. 1997a, ApJ, 480, L111

Usov, V. V. 1998, Phys. Rev. Lett., 80, 230

Usov, V. V. 2001, Phys. Rev. Lett. 87, 021101

Wang, Q. D., \& Lu, T. 1984, Phys. Lett., B148, 211

Weber, F. 1999, J. Phys. G: Nucl. Part. Phys., 25, R195

Witten, E., 1984, Phys. Rev., D30, 272

Xu, R.X. 2002, ApJ, 570, L65

Xu, R. X., \& Qiao, G. J. 1998, Chin. Phys. Lett., 15, 934

Xu, R. X., \& Qiao, G. J. 1999, Chin. Phys. Lett., 16, 778

Xu, R.X., Qiao, G.J., Zhang, B., 1999, ApJ, 522, L109

Xu, R. X., Zhang, B., Qiao, G. J. 2001, Astropart. Phys., 15, 101

Zhang, B., Xu, R. X, Qiao, G. J. 2000, ApJ, 545, L127 